\title{
Therapie von Long-Covid-Syndromen mit chinesischer Arzneimitteltherapie im Erwachsenen-, Kindes- und Jugendalter
}

\author{
Christian Thede ${ }^{1}$ \\ Online publiziert: 7. Oktober 2021 \\ (c) Springer Medizin Verlag GmbH, ein Teil von Springer Nature 2021
}

\section{Zusammenfassung}

Chronische Folgeerkrankungen nach überstandener Covid-19-Erkrankung und auch nach inapparenten bzw. symptomarmen SARS-CoV-2-Infektionen stellen trotz der wachsenden Zahl geimpfter Personen und deutlich reduzierter Infektionsraten im Sommer 2021 ein zunehmend häufiges Problem dar. Für diese an „Long-Covid-Syndrom“ Erkrankten gibt es bisher im Bereich der westlichen Medizin kaum etablierte wirksame Therapiemöglichkeiten. Im vorliegenden Artikel werden effektive Therapiekonzepte der chinesischen Arzneimitteltherapie zur Behandlung der besonders häufigen respiratorischen und neurologischen Symptomatik sowie von „Chronic-Fatigue-Syndromen“ (CFS) vorgestellt, unter besonderer Berücksichtigung der Behandlung spezieller Formen von Covid-19-Folgezuständen als Dysharmonie des yang minor (Kleines Yang, shaoyang) mit Variationen des „Kleinen Bupleurum-Dekokts“ (Xiao chaihu tang), auch im Hinblick auf mögliche Reaktivierungen chronischer EBV-Infektionen durch Corona-Virus-Infektionen. Des Weiteren werden die Bedeutung von Long-Covid bei Kindern und Jugendlichen erörtert und mögliche Therapiekonzepte skizziert.

Schlüsselwörter Covid-19 · SARS-CoV-2 · Long-Covid · Post-Covid · Chronic-Fatigue-Syndrom (CFS) · Chinesische Arzneimitteltherapie · Yang-minor-Syndrom (Kleines Yang, Shaoyang) • „Kleines Bupleurum-Dekokt“ (Xiao chaihu tang) $\cdot$ Ebstein-Barr-Virus-Infektion $\cdot$ EBV

\section{Chinese herbal medicine in the therapy of post-covid syndromes in adults, children and adolescents}

\begin{abstract}
Chronic sequelae after surviving Covid 19 disease, and also after inapparent or asymptomatic SARS-CoV-2 infections, represent an increasingly common problem despite the growing number of vaccinated individuals and significantly reduced infection rates in the summer of 2021. For these sufferers of "post-Covid syndrome" Western medicine has been unable to date to offer any significant, established and effective therapy options. In this article, the author presents effective therapeutic concepts offered by Chinese herbal therapy for the treatment of the particularly frequent respiratory and neurological symptoms as well as for "chronic fatigue syndrome" (CFS), with special emphasis on the treatment of special forms of Covid-19 sequelae such as disharmony of lesser yang (shaoyang) with variations of "minor bupleurum decoction" (xiao chaihu tang), also with regard to possible reactivations of chronic EBV infections by corona-virus infections. The article also discusses the significance of Long Covid in children and adolescents and outlines possible therapeutic concepts. The article concludes with two case studies.
\end{abstract}

Keywords Covid-19 · SARS-CoV-2 · Long-Covid · Post-Covid · Chronic-Fatigue Syndrome (CFS) · Chinese herbal therapy · Lesser yang Syndrome (shaoyang) · "Minor Bupleurum Decoction" (Xiao chaihu tang) ·

Ebstein-Barr-Virus-Infection · EBV

Christian Thede

info@praxis-thede.de

1 Gorch-Fock-Weg 16, 23730 Neustadt/Holstein, Deutschland 


\section{Einleitung}

Sowohl nach überstandenen Covid-19-Erkrankungen als auch nach symptomarmen oder inapparenten Infektionen mit SARS-CoV-2 treten bei einem hohen Prozentsatz der Betroffenen (RKI 11/2020: etwa 40\% (RKI 2020)) Folgezustände auf, für die sich nach diversen alternativen Vorschlägen die Bezeichnung (Datta et al. 2020; Baig 2020) „Long-Covid“ durchzusetzen scheint. Unsicherheiten bestehen noch über die Pathophysiologie, die der beobachteten heterogenen Symptomatik zugrunde liegt. Die Einschätzungen der tatsächlichen Häufigkeit von LongCovid-Symptomatik liegen aktuell eher bei 10-20\%, fallen jedoch angesichts einer möglicherweise hohen Dunkelziffer und der schwierigen Zuordnung auftretender Beschwerden sehr unterschiedlich aus. Außerdem ist noch nicht geklärt, inwieweit Impfungen, die zwar in einem hohen Prozentsatz vor ernsthaften Covid-19-Erkrankungen schützen, jedoch insbesondere bei den mutierten Virusvarianten symptomarme oder blande Infektionen mit SARS-CoV-2 deutlich seltener verhindern können, der Entwicklung einer LongCovid-Symptomatik wirksam vorbeugen können.

Am häufigsten werden Schwächezustände beschrieben, die dem bereits bekannten Chronic-Fatigue-Syndrom ähneln, gefolgt von Atemnot, Husten und depressiven $\mathrm{Zu}$ ständen (Manal et al. 2020; Carfi et al. 2020). Häufige persistierende Störungen sind außerdem kognitive und sensorische Defizite, ebenso Befunde wie persistierend erhöhte Werte für D-Dimere, C-reaktives Protein (CRP) und pathologische Röntgenbefunde. Seltener, jedoch bedrohlich, sind Komplikationen der mit Covid-19 assoziierten Endothelitis wie embolische Prozesse und Vaskulitiden. Die auftretenden Störungen halten vielfach mehrere Monate an. Außerdem mehren sich Berichte über das im Kindesalter auftretende „Pediatric Inflammatory Multisystem Syndrome“ (PIMS).

Therapieoptionen zur Behandlung akuter Covid-19-Erkrankungen mit chinesischer Arzneimitteltherapie (CAM) sind vom Autor der vorliegenden Arbeit in mehreren Veröffentlichungen bereits eingehend diskutiert worden (Thede 2020a, b, c; Thede et al. 2020). Angesichts der zunehmenden Bedeutung von Long-Covid-Erkrankungen wird der Fokus dieser Arbeit auf der Diskussion möglicher Therapieoptionen mit CAM bei Folgezuständen von Covid-19 - unter besonderer Berücksichtigung von Fatigue-Symptomatik, depressiven Episoden sowie kognitiven und sensorischen Defiziten - liegen.

\section{Covid-19 aus Sicht der Chinesischen Medizin - pathogenetische Bedingungen}

Zum Verständnis von Störungen in der "Post-Covid-Phase“ ist die Kenntnis der pathophysiologischen Entwicklungen in der Akutphase von Bedeutung, die hier kurz rekapituliert werden.

\subsection{Das infektiöse Pathogen}

Über die Identifizierung von ,epidemischem humor-Toxischem(,Feuchtigkeit"-Toxischem, shidu)" als dominierendem pathogenem Faktor besteht bereits seit frühen Veröffentlichungen aus China (National Health Commission of the PRC und National Administration of Traditional Chinese Medicine of the PRC 2020; Zhao et al. 2020; Zhong et al. 2020; Zhao and Flanagan 2020) allgemeiner Konsens.

\subsection{Frühe Phase der Infektion}

Initial verläuft die Infektion mit SARS-CoV-2 oft mild oder symptomlos. Die frühe Symptomatik kann sehr unterschiedlich sein und ist meist nicht eindeutig den in der Chinesischen Medizin (CM) bekannten Krankheitskategorien für Infektionserkrankungen der Atemwege - algor laedens (,schädigende Kälte“, shanghan) bzw. morbi temperati (,Wärme“-Erkrankungen, wenbing) - zuzuordnen. Dementsprechend wird auch in dieser Phase die Erkrankung als Folge der Affektion durch epidemisches humorToxisches („Feuchtigkeit“-Toxisches, shidu) - unter Berücksichtigung evtueller Pathogene wie ventus (,Wind“, feng), algor (,Kälte“, han) und calor („Hitze“, re) - betrachtet und therapiert.

\subsection{Covid-19 - respiratorische Symptomatik}

Die akute pulmonale Symptomatik mit Dyspnoe, Husten, evtl. Schleimexpektoration, oft mit Allgemeinsymptomen wie Fieber, Schwäche und Beschwerden der Verdauung wie Appetitlosigkeit, Übelkeit, Diarrhoe bzw. stockender Verdauung einhergehend, ist als Affektion der oo. pulmonalis, lienalis et stomachi (Fk „Lunge“ und „Mitte“/Fk „Milz“ und „Magen“, fei pi wei) zu verstehen. In der Folge werden die Transformation durch das Qi des o. lienalis (Fk „Milz“, pi) sowie die Transportfunktionen des qi pulmonale et stomachi (Qi der Fk „Lunge“ und „Magen“, fei wei qi) blockiert, was zu weiterer Akkumulation von pathologischem humor („Feuchtigkeit“, shi) bzw. pathologischer pituita (,Schleim“, tan) führt, wodurch sich aus Sicht der Chinesischen Medizin die Überladung von Interstitium und Alveolen und im weiteren Verlauf die beobachteten Hyperimmunreaktionen erklären. 


\subsection{Weitere energetische Entgleisungen bei komplizierten und schwerwiegenden Verläufen}

- Die respiratorische Insuffizienz, z. T. mit der Notwendigkeit maschineller Beatmung als Ausdruck des kompletten Zusammenbruchs des Qi-Mechanismus des o. pulmonalis (Fk „Lunge“, fei).

- Im weiteren Krankheitsverlauf können sich sogenannte toxische Blockaden entwickeln, die zum einen zur Entwicklung von gravierenden calor-Befunden („Hitze“, re) führen, mit oft schweren septischen Krankheitsverläufen, zum anderen in Xue-Stasen münden, mit der Folge diverser Herz-Kreislauf-Komplikationen (Thede 2020b, c; Thede et al. 2020).

\subsection{Residualzustände als Folge postakuter energetischer Entgleisungen}

- Depletio qi (energetische Schwäche des Qi, qixu) der oo. pulmonalis et lienalis (Fk „Lunge“ und „Milz“, fei pi) können Ergebnis der Affektion und Akkumulation durch humor-Pathogene („Feuchtigkeit“, shi) im Rahmen von Covid-19-Erkrankungen sein. Über diesen Mechanismus ist auch die mögliche Akkumulation von pituita („Schleim“, tan) zu erklären.

- Nach Überstehen der Akuterkrankung können Xue-Stasen persistieren und zu verschiedenen Gefäß- und Entzündungskomplikationen führen, deren mögliche Therapie mit Chinesischer Medizin bereits an anderer Stelle beschrieben worden ist (Thede 2020b, c; Thede et al. 2020).

- Der oben beschriebene calor-Prozess („Hitze“, re) während der Akuterkrankung kann zu Säfte-Verlust und depletio yin (energetische Schwäche des Yin, yinxu) führen.

\section{Long-Covid - Differenzierung der Residualzustände in der Chinesischen Medizin (CM)}

In der Rekonvaleszenzphase nach einer SARS-CoV-2-Infektion sind vor allem folgende Symptomkomplexe von Bedeutung:

- Respiratorische Störungen wie (Belastungs-)Dyspnoe und Husten,

- Chronic-Fatigue-Syndrome (CFS) mit Müdigkeit und Schwächezuständen,

- depressive Episoden,

- neurologische Störungen wie kognitive und sensorische Defizite,
- Gefäßerkrankungen wie Apoplexien, Thromboembolien und Vaskulitiden (Sollini et al. 2020).

\subsection{Respiratorische Symptome}

Häufigste Symptome sind Atemnot und Husten - nicht nur nach schweren Covid-Pneumonien, sondern oft auch nach milden Verläufen. Aus der Perspektive der Chinesischen Medizin ist hier in erster Linie an folgende Entgleisungen zu denken:

\subsubsection{Depletio qi (energetische Schwäche des $\mathrm{Qi}$, qixu) der

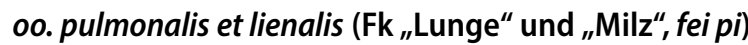

Typische Symptome Spontane Atemnot bzw. Kurzatmigkeit bei leichten Anstrengungen, persistierender Husten, Tagschweiße, Müdigkeit und Schwäche sowie eine blasse Zunge.

Therapiemöglichkeiten Bei überwiegender depletio (energetische Schwäche, $x u$ ) des qi pulmonale (Qi des Fk „Lunge“, feiqi) kann besonders bei älteren Betroffenen die Rezeptur „Dekokt zur Stützung des Fk Lunge (o. pulmonalis)“ (Bufei tang, s. Scheid et al. (2009, S. 331)), evtl. mit Modifikationen, als Basisrezeptur zur Rekonstitution der Qi-Funktionen und der Säfte des o. pulmonalis (Fk „Lunge“, fei) dienen:

- Ginseng radix (Renshen)

- Astragali radix (Huangqi)

- Rehmanniae radix praeparata (Shudihuang)

- Schisandrae fructus (Wuweizi)

- Asteris radix (Ziwan)

- Mori cortex (Sangbaipi)

Für ein kombiniertes depletio qi (energetische Schwäche des Qi, qixu) der oo. pulmonalis et lienalis (Fk ,Lunge“ und „Milz“, fei pi) sind Modifikationen folgender Rezeptur geeignet: „Dekokt der sechs Edlen“ (Liu junzi tang, s. Hempen et al. (2006, S. 336))

- Ginseng radix (Renshen)

- bzw. Codonopsis radix (Dangshen)

- Atractylodis macrocephalae rhizoma (Baizhu)

- Poria (Fuling)

- Glycyrrhizae radix (Gancao)

- Citri reticulatae pericarpium (Chenpi)

- Pinelliae rhizoma (Banxia) 


\subsubsection{Kombinierte depletio (energetische Schwäche, $x u$ ) von Qi und Yin}

Symptome Über Kurzatmigkeit und Schwächesymptomatik hinausgehend: trockener Husten mit weiteren Trockenheitszeichen und evtl. subfebrile Temperaturen bei gerötetem, trockenem Zungenkörper

Therapie Bei vorwiegender depletio yin (energetische Schwäche des Yin, yinxu) sind Modifikationen der Rezeptur "Glehnia- und Ophiopogon-Dekokt“ (Shashen maidong tang, s. Scheid et al. (2009, S. 669)) angezeigt:

- Glehniae radix (Beishashen)

- Ophiopogonis radix (Maimendong)

- Polygonati odorati rhizoma (Yuzhu)

- Mori folium (Sangye)

- Trichosanthis radix (Tianhuafen)

- Lablab semen album (Biandou)

- Glycyrrhizae radix (Gancao)

Wenn außerdem die o.g. Anzeichen einer depletio qi (energetische Schwäche des Qi, qixu) wesentliche Bestandteile der Symptomatik sind, können Kombinationen dieser Rezeptur mit Teilen der beiden zuvor genannten zur Therapie einer depletio qi (energetische Schwäche des Qi, qixu) in Erwägung gezogen werden.

Cave Trichosanthis radix (Tianhuafen) nicht bei Frauen im gebärfähigen Alter anwenden.

\section{Modifikationen}

- Bei ausgeprägter Schmälerung der Säfte mit Panacis quinquefolii radix (Xiyangshen), Adenophorae radix (Nanshashen), Schisandrae fructus (Wuweizi)

- Bei ausgeprägtem Husten ohne Expektorat mit Armeniacae semen (Xingren), Stemonae radix (Baibu), Asteris radix (Ziwan)

- Bei Husten mit spärlichem zähem Schleim mit Trichosanthis semen (Gualouren), Fritillariae cirrhosae bulbus (Chuanbeimu)

\subsubsection{Persistierender pituita-Befund („Schleim“, tan)}

Die häufigsten pathogenetischen Wege, die postakut zur Entstehung eines persistierenden pituita-Befunds („Schleim“, tan) beitragen können, sind folgende:

- Eine persistierende Blockade der Qi-Transformation in der „Mitte“ und der Transportfunktion des qi pulmonale (Qi des Fk „Lunge“, feiqi) führt zu pituita algida (,KälteSchleim“, hantan).
- Ein calor-Prozess („Hitze“, re) während der akuten Covid-19-Erkrankung führt über das Eintrocknen von Säften zu pituita calida (,Hitze-Schleim“, retan).

\section{Therapie je nach Differenzierung:}

\subsubsection{Pituita algida („Kälte-Schleim“, hantan) blockiert den o. pulmonalis (Fk "Lunge“, fei)}

Leitsymptomatik Husten mit farblosem Schleimauswurf sowie evtl. Atemnot, thorakales Druck- bzw. Engegefühl, Verschlimmerung im Liegen bei typischerweise geschwollener blasser Zunge mit feuchtem weißem Belag

Mögliche Grundrezeptur zur Stützung und Harmonisierung der "Mitte" sowie zur Umwandlung und Eliminierung von pituita („Schleim“, tan) „Dekokt der sechs Edlen“ (Liu junzi tang, s. oben).

\section{Modifikationen}

- Zur Verstärkung von Hustenstillung bzw. Schleimlösung mit Armeniacae semen (Xingren), Platycodi radix (Jiegeng), Magnoliae cortex (Houpo)

- Zur Hustenstillung, Schleimlösung und Lösung von persistierenden Verdauungsblockaden - besonders bei älteren Erkrankten - mit dem „Dekokt der drei Samen zur Erhaltung der Eltern“ (San zi yang qin tang, s. Hempen et al. (2006, S. 408)): Perillae fructus (Zisuzi), Sinapis albae semen (Baijiezi), Raphani semen (Laifuzi)

\subsubsection{Pituita calida („Hitze-Schleim“, retan)}

Zeichen und Symptome Dyspnoe, thorakales Druckgefühl sowie Husten mit gelbem bzw. gelb-grünem, oft zähem Expektorat bei geröteter Zunge mit gelbem Belag

Zwei mögliche Therapieprinzipien Zur Hustenstillung und Lösung des pituita-Befunds (,Schleim“, tan) durch Kühlung und Befeuchtung der Säfte: „Pulver mit Fritillaria und Trichosanthes“ (Beimu gualou san, s. Hempen et al. (2006, S. 256)) als Basisrezeptur:

- Fritillariae cirrhosae bulbus (Chuanbeimu)

- Trichosanthis fructus (Gualou)

- Trichosanthis radix (Tianhuafen)

- Poria (Fuling)

- Citri reticulatae pericarpium (Chenpi)

- Platycodi radix (Jiegeng)

Cave Trichosanthis radix (Tianhuafen) ist bei Frauen im gebärfähigen Alter kontraindiziert. 


\section{Modifikationen}

- Bei gering ausgeprägter Schmälerung der Säfte und zur Akzentuierung der Schleimlösung Fritillariae thunbergii bulbus (Zhebeimu) statt Fritillariae cirrhosae bulbus (Chuanbeimu)

- Bei starkem Hustenreiz mit Armeniacae semen (Xingren), Eriobotryae folium (Pipaye)

Ein alternatives Konzept zur Lösung des Schleimbefunds, das die Methode des kühlenden Befeuchtens mit jener der Umwandlung durch scharfe Arzneien kombiniert, wird durch Modifikationen der Rezeptur „Pille, die das Qi klärt und Schleim transformiert" (Qingqi huatan wan, s. Hempen et al. (2006, S. 254)) verwirklicht:

- Pinelliae rhizoma (Banxia)

- Citri reticulatae pericarpium (Chenpi)

- Poria (Fuling)

- Glycyrrhizae radix (Gancao)

- Scutellariae radix (Huangqin)

- Trichosanthis semen (Gualouren)

- Arisaematis rhizoma (Tiannanxing)

- Armeniacae semen (Xingren)

- Aurantii fructus immaturus (Zhishi)

- Zingiberis rhizoma recens (Shengjiang)

\section{Modifikationen}

- Zur Hustenstillung mit Platycodi radix (Jiegeng), Eriobotryae folium (Pipaye)

- Zur verstärkten Lösung zähen gelben Schleims mit Fritillariae thunbergii bulbus (Zhebeimu)

\subsubsection{Xue-Stasen}

Nicht nur bei Gefäßkomplikationen, sondern auch bei restriktiven Ventilationsstörungen im Gefolge einer SARSCoV-2-Infektion können Xue-Stasen eine wichtige Rolle spielen und unter Umständen in Kombination mit den o.g. Störungen auftreten. Hinweisende Zeichen und Symptome können sein: Belastungsdyspnoe, thorakales Druckgefühl, livide Verfärbungen der Zunge bzw. gestaute Unterzungenvenen, p. asper (,rauer“ Puls, se mai).

Zur Lösung der Xue-Stasen, der Befreiung des energetischen Flusses im Brustraum und der Bereinigung persistierender entzündlicher Tendenzen ist folgende Rezeptur bzw. deren Modifikationen in Betracht zu ziehen: „Stasen aus der Versammlungshalle des Xue vertreibendes Dekokt" (Xuefu zhuyu tang, s. Hempen et al. (2006, S. 298))

- Persicae semen (Taoren)

- Carthami flos (Honghua)

- Angelicae sinensis radix (Danggui)

- Chuanxiong rhizoma (Chuanxiong)
- Paeoniae radix rubra (Chishaoyao)

- Achyranthis bidentatae radix (Niuxi)

- Bupleuri radix (Chaihu)

- Platycodi radix (Jiegeng)

- Aurantii fructus (Zhike)

- Rehmanniae radix (Shengdihuang)

- Glycyrrhizae radix (Gancao)

Anmerkung In der Schwangerschaft kontraindiziert

\section{Weitere für diese Problematik geeignete Arzneimittel sind}

- Zur Lösung von Xue-Stasen u. a. im Thorax: Salviae miltiorrhizae radix (Danshen), Corydalidis rhizoma (Yanhusuo), Curcumae longae tuber (Yujin), Olibanum (Ruxiang)

- Zur Befreiung der Qi-Zirkulation im Thorax evtl. in Kombination mit dem „Dekokt mit Trichosanthis fructus, Allii bakeri bulbus und Pinelliae rhizoma“ (Gualou xiebai banxia tang, s. Scheid et al. (2009, S. 516)): Trichosanthis fructus (Gualou), Allii macrostemonis bulbus (Xiebai), Pinelliae rhizoma (Banxia)

- Zur Lösung von thorakaler Qi-Stagnation und Xue-Stasen alternativ oder in Kombination mit dem ,SalviaTrank“ (Danshen yin, s. Hempen et al. (2006, S. 296)): Salviae miltiorrhizae radix (Danshen), Amomi xanthioidis fructus (Sharen), Santali albi lignum (Tanxiang)

\subsection{Chronic-Fatigue-Syndrome (CFS) und andere Schwächezustände}

Vorwiegend Erwachsene leiden auch mehrere Monate nach einer überstandenen symptomatischen oder symptomarmen SARS-CoV-2-Infektion noch an Beschwerden, die an Chronic-Fatigue-Syndrome (CFS) erinnern. Häufig wird von z.T. plötzlich auftretenden Schwächezuständen, Müdigkeit, Antriebsarmut sowie sensorischen und kognitiven Defiziten (,brain fog“) und Schmerzzuständen berichtet. Die Symptome führen nicht selten zu längerer Arbeitsunfähigkeit bzw. dem Verlust von Alltagskompetenzen. Während neben der Krankheitsbezeichnung international noch Klassifizierungen und pathogenetische Wege kontrovers diskutiert werden, besteht weitgehende Einigkeit in der Literatur, dass CFS als eine meist nach Virusinfektionen auftretende Multisystemerkrankung anzusehen und durch neuroimmunologische Dysregulation und Störungen des Energiestoffwechsels gekennzeichnet ist.

Da technische Befunde wie veränderte Entzündungsparameter oder Organbefunde in dieser Phase in der Regel nicht nachweisbar sind, fehlen im Bereich der westlichen Medizin angesichts unklarer Ätiologie gesicherte Therapiekonzepte. 
Besonders häufig nach SARS-CoV-2-Infektionen geschilderte CFS-Symptome sind

- Schwäche: Oft konstant erniedrigtes Energieniveau, aber auch Schwächeattacken, die z.T. durch physische bzw. emotionale Belastungen getriggert werden - charakteristisch dabei: Das Ausmaß der Erschöpfung (oft auch die Verschlimmerung weiterer Symptome) ist deutlich gravierender, als es der jeweilige Trigger normalerweise erwarten ließe (PENE - ,post exertional neuroimmune exhaustion").

- Müdigkeit am Tage, oft bei zugleich auftretenden Schlafstörungen in der Nacht,

- Antriebsarmut, depressive Symptomatik,

- vegetative Symptomatik wie Herz-Kreislauf-Dysregulation, Störungen der Verdauung, des Appetits, der Schweißsekretion und des Temperaturempfindens,

- diffuse, z. T. wandernde Gelenk- und Muskelschmerzen,

- neurologische Störungen: kognitive und sensorische Defizite.

Da eine Reihe der genannten Symptome auch bei depressiven Episoden und bei Fibromyalgie vorkommen, wird nicht wenigen an Long-Covid-Erkrankten eine dieser Diagnosen „gegeben“. Die sich daraus ergebenden Therapiestrategien werden allerdings aus Sicht des Autors dieses Artikels der postinfektiösen Genese der Symptomatik der Betroffenen nicht gerecht, weil sie die tatsächliche pathogenetische (postinfektiöse) Entwicklung unberücksichtigt lassen. Davon unberührt bleibt, dass Menschen nach einer akuten Covid-19-Erkrankung oder im Laufe einer Long-CovidErkrankung eine behandlungsbedürftige Depression entwickeln können.

Aus der Sicht der Chinesischen Medizin treten im Rahmen von Schwächezuständen bei an Long-Covid-Leidenden häufig folgende Störungsmuster auf:

\subsubsection{Depletio qi (energetische Schwäche des Qi, qixu) der

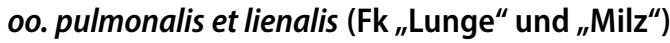

Neben der allgemeinen Müdigkeit und Schwäche sind hier typischerweise Schwere- bzw. Schwächegefühle der Extremitäten, Antriebsarmut, depressive Zustände, schnelle Erschöpfbarkeit mit kurzzeitiger Erholung durch Pausen zu beobachten, häufig in Kombination mit humor-Symptomen (,Feuchtigkeit“, shi) durch die mangelnde Transformation im Rahmen der depletio qi (energetische Schwäche des Qi, qixu), wozu auch persistierender Geschmacks- und Geruchsverlust gehören können.

Bei dieser Konstellation sind Arzneimittelkonzepte, die auf der Grundrezeptur „Dekokt der sechs Edlen“ (Liu junzi tang, s. oben) aufbauen, angezeigt.

Alternativ kann als Basisrezeptur das „Pulver mit Ginseng, Poria und Atractylodis macrocephalae“ (Shenling baizhu san, s. Hempen et al. (2006, S. 338)) verwendet werden:

- Ginseng radix (Renshen)

- Atractylodis macrocephalae rhizoma (Baizhu)

- Poria (Fuling)

- Glycyrrhizae radix (Gancao)

- Dioscoreae rhizoma (Shanyao)

- Lablab semen album (Biandou)

- Nelumbinis semen (Lianzi)

- Coicis semen (Yiyiren)

- Amomi xanthioidis fructus (Sharen)

- Platycodi radix (Jiegeng)

Selbstverständlich können auch Kombinationen mit vorbestehenden oder im Verlauf der Erkrankung erworbenen Störungsmustern wie depletio xue (energetische Schwäche des Xue, хиехи) oder Stagnation des qi hepatici (Qi des Fk „Leber“, ganqi) die Situation komplizieren und müssen bei der Erstellung der Behandlungskonzepte berücksichtigt werden.

\subsubsection{Kombinierte depletio (energetische Schwäche, $x u$ ) von Qi und Yin}

Bei einer kombinierten depletio (energetische Schwäche, $x u$ ) von Qi und Yin kann nicht nur trockener Husten (siehe oben) die Symptomatik prägen, sondern auch eine Müdigkeits- und Schwächesymptomatik dominieren. Therapeutisch kommen mit entsprechenden Modifikationen die o.g. Konzepte (,Respiratorische Symptome: Kombinierte depletio (energetische Schwäche, $x u$ ) von Qi und Yin“) in Frage.

\subsection{Störungen des Geruchs- und Geschmackssinns sowie Schwindel und kognitive Defizite}

Störungen des Sensoriums werden in der Chinesischen Medizin oft als Ansammlung von humor („,Feuchtigkeit“, shi) bzw. pituita (,Schleim“, tan) identifiziert, wodurch die Sinnesöffnungen blockiert werden. In Ergänzung zu einer den Grundbefund behandelnden Rezeptur - z. B. dem „Dekokt der sechs Edlen“ (Liu junzi tang, s. oben) bei einer depletio qi (energetische Schwäche des Qi, qixu) - bieten sich hier besonders aromatische Arzneien zur Umwandlung der humor-Störung („Feuchtigkeit“, shi) an wie Acori rhizoma (Shichangpu), Agastachis herba (Huoxiang) sowie Arzneimittel zur Klärung des Sensoriums wie Polygalae radix (Yuanzhi) und Polygoni multiflori caulis (Yejiaoteng). Zur Klärung von Xue-Stasen - die einem entzündlichen Aspekt dieses Geschehens entsprechen - kann bei dieser Problematik Curcumae longae tuber (Yujin) eine wertvolle Ergänzung sein.

Kognitive Defizite wie Denk- und Konzentrationsstörungen und Wortfindungsstörungen sind meist ebenfalls als 
Akkumulation trüber pituita (trüber „Schleim“, zhuotan) anzusehen - die die Funktionen der ,Mitte“ blockiert bzw. die Sinnesöffnungen (die „Poren des Herzens“) verlegt und sind somit auf gleiche Weise behandelbar.

\section{Verwendung des „Kleines Bupleurum- Dekokts" (Xiao chaihu tang) bei Chronic- Fatigue-Syndromen (Thede 2021)}

Dysharmonie des yang minor (Kleines Yang, shaoyang) Schon im 6. Kapitel des „Inneren Klassikers des Gelben Fürsten“" (Huangdi Neijing, aus dem 1. Jh. v.u.Z., im 8. und 11. Jh. überarbeitet) wurde als Voraussetzung der harmonischen Funktion des öffnenden (yang maior, Großes Yang, taiyang) und schließenden (splendor yang, Überstrahlung des Yang, yangming) Prinzips die besondere Bedeutung der angelpunktartigen Funktion des yang minor (Kleines Yang, shaoyang) betont. Eine Störung im yang minor (Kleines Yang, shaoyang) kann einerseits die Regulation zwischen dem Inneren und Äußeren im Organismus und andererseits die des Aufsteigens des ,klaren Qi“ und des Absenkens des „Trüben“ behindern. Außerdem können eingedrungene Pathogene nicht effektiv eliminiert werden. Die häufig resultierende Symptomatik ist gekennzeichnet durch Dysregulation von Wärme und Kälte (plötzlich wechselndes Temperaturempfinden, bitterer Mundgeschmack, Halstrockenheit), durch blockierte bzw. geschwächte Funktionen der „Mitte“ (Appetitverlust, Völlegefühl, Übelkeit, evtl. Schwäche, Antriebslosigkeit) und durch gestörte Transformation von Flüssigkeiten mit Akkumulation von pituita (,Schleim“, tan) bzw. humor (,Feuchtigkeit“, shi) (Schwindel, feuchter Zungenbelag) sowie durch Stagnationen des Qi (Spannungsgefühle, chordale (,saitenförmige“, xian) Pulsqualität).

In diesen Fällen ist die in der ,Abhandlung über schädigende Kälte (algor laedens)“ (Shanghan lun, von Zhang Zhongjing, aus dem 2. Jh.) erstmals genannte Rezeptur „Kleines Bupleurum-Dekokt“ (Xiao chaihu tang, s. Hempen et al. (2006, S. 156)) angezeigt:

- Bupleuri radix (Chaihu)

- Scutellariae radix (Huangqin)

- Pinelliae rhizoma (Banxia)

- Zingiberis rhizoma recens (Shengjiang)

- Ginseng radix (Renshen)

- Glycyrrhizae radix (Gancao)

- Jujubae fructus (Dazao)

Anmerkungen Ginseng radix (Renshen) wird häufig ersetzt durch Codonopsis radix (Dangshen), Kontraindikationen siehe unter „Entscheidungskriterien“ (s. unten).

Die Rezeptur wird im Ursprungstext zwar zunächst im
Kontext verschiedener Stadien des Verlaufs einer Erkrankung durch algor-Pathogene (,Kälte“, han) angegeben, doch sind seitdem diverse weitere mögliche Indikationen für die Rezeptur diskutiert worden. So wurde auch für die akute Covid-19-Erkrankung bereits - auch wenn die zumeist beobachtete Klinik nicht dem typischen Verlauf einer Affektion durch algor-Pathogene (,Kälte“, han) entspricht - die Anwendung des ,Kleinen Bupleurum-Dekokts" (Xiao chaihu tang) vorgeschlagen. Das epidemische humor-Pathogen (,Feuchtigkeit“, shi) beeinträchtigt offensichtlich auch die o.g. Funktionen des yang minor (Kleines Yang, shaoyang), und somit ergibt sich unter Umständen die Notwendigkeit der yang-minor-Harmonisierung (Kleines Yang, shaoyang).

Die immer häufiger bei Long-Covid-Patienten beschriebene Schwäche- und Müdigkeitssymptomatik (ChronicFatigue-Syndrom/CFS) zeigt auffällige Ähnlichkeiten zu CFS-Verläufen nach Infektionen mit dem Ebstein-BarrVirus (EBV) (Thede 2020c, 2021). In einer kleinen Studie fanden sich bei 20 von 30 Probanden mit Long-Covid-Symptomatik Antikörper-Titer, die auf eine Reaktivierung einer vorbestehenden EBV-Infektion hinweisen. Wenngleich diese Befunde noch bestätigt werden müssen, werfen sie die Frage auf, ob Long-Covid-Symptomatik zumindest teilweise als Folge einer Reaktivierung einer zurückliegenden EBV-Infektion zu verstehen ist (Gold et al. 2021). Das könnte eine mögliche Erklärung für die oft frappierende Ähnlichkeit der beobachteten CFS-Symptomatik bei LongCovid-Erkrankten einerseits und an chronischen EBVInfektionen Leidenden andererseits liefern.

Der Autor dieses Artikels kann auf zahlreiche Behandlungsverläufe zurückblicken, in denen die CFS-Symptomatik nach EBV-Infektionen durch die Anwendung von Modifikationen des „Kleinen Bupleurum-Dekokts“ (Xiao chaihu tang) regelmäßig komplett und nachhaltig geheilt werden konnte. Da mehrere der Betroffenen Leistungssport treiben, konnte in diesen Fällen die Wiederherstellung der Leistungsfähigkeit durch Leistungsdiagnostik eindrucksvoll verifiziert werden.

Dieses Konzept ist nach den Erfahrungen des Autors nicht nur bei EBV-Infektionen ausgesprochen effektiv, sondern es konnte auch im Fall von Long-Covid-Erkrankungen auf diese Weise in mehreren Fällen Beschwerdefreiheit erreicht werden, was mit der möglichen Assoziation von SARS-CoV-2- und EBV-Infektionen in Zusammenhang stehen könnte. Die Erhebung eines EBV-Antikörperstatus ist vor diesem Hintergrund bei an Long-Covid Erkrankten zu empfehlen.

Bei der Anwendung des „Kleinen Bupleurum-Dekokts“ (Xiao chaihu tang) ist zu beachten und darüber aufzuklären, dass vorübergehend eine mäßig ausgeprägte, an einen frischen Infekt erinnernde Symptomatik (z. B. subfebrile Temperaturen, Hitzeempfinden, Schnupfen, Schleimhusten) 
auftreten kann, was als Restitution der Regulation des Öffnenden und Schließenden (s. oben) und somit der Elimination des pathologischen Prozesses anzusehen ist. Diese Nebenwirkung stellt also eine Begleiterscheinung des erwünschten therapeutischen Effekts dar, was in der Regel daran erkennbar ist, dass das Phänomen mit einer Verbesserung des Allgemeinzustands und der Kraftreserven einhergeht.

Von den zahlreichen möglichen Modifikationen dieser Rezeptur seien hier einige genannt, die für Long-CovidSymptomatik besonders geeignet sind:

- Bei Dyspnoe mit Trichosanthis fructus (Gualou), Platycodi radix (Jiegeng)

- Bei Schleimhusten mit Magnoliae cortex (Houpo), Belamcandae rhizoma (Shegan) oder Platycodi radix (Jiegeng), Trichosanthis fructus (Gualou), Fritillariae thunbergii bulbus (Zhebeimu)

- Bei trockenem Husten mit Schisandrae fructus (Wuwei$z i$ ), Fritillariae cirrhosae bulbus (Chuanbeimu), Armeniacae semen (Xingren)

- Bei humor-Symptomatik (,Feuchtigkeit“, shi) mit Magnoliae cortex (Houpo), Atractylodis rhizoma (Cangzhu), Poria (Fuling)

- Bei ausgeprägtem Völlegefühl mit Aurantii fructus immaturus (Zhishi), Citri reticulatae pericarpium (Chenpi)

- Bei Halsschmerzen mit Lonicerae flos (Jinyinhua), Forsythiae fructus (Lianqiao)

- Bei sensorischen Defiziten und mentalen Störungen mit Acori rhizoma (Shichangpu), Agastachis herba (Huoxiang), Polygalae radix (Yuanzhi), Polygoni multiflori caulis (Yejiaoteng)

- Bei Anzeichen für Xue-Stasen siehe Abschnitt „Xue-Stasen“

Das beschriebene Therapiekonzept hat sich nach Erfahrungen des Autors als ähnlich hocheffektiv erwiesen wie bei Chronic-Fatigue-Syndromen (CFS) im Rahmen chronischer Infektionen mit dem Ebstein-Barr-Virus (EBV), wobei der o.g. mögliche Zusammenhang zwischen den Infektionen mit SARS-CoV-2 und EBV zu berücksichtigen ist und noch weiterer Klärung bedarf.

\section{Entscheidungskriterien für die Anwendung des „Kleinen Bupleurum- Dekokts" (Xiao chaihu tang) bei Long- Covid-Erkrankungen}

- Andauernde oder neu aufgetretene CFS-Symptomatik nach gesicherten oder vermuteten SARS-CoV-2-Infektionen.

- Hinweise auf eine vorbestehende Infektion mit EBV.
- Auftreten typischer yang-minor-Symptome (Kleines Yang, shaoyang), wobei bereits in der „Abhandlung über schädigende Kälte (algor laedens)" (Shanghan lun) darauf hingewiesen wird, dass unter Umständen ein einziges typisches Symptom auf ein yang-minor-Syndrom (Kleines Yang, shaoyang) hinweisen und eine Indikation für das „Kleine Bupleurum-Dekokt“ (Xiao chaihu tang) bedeuten kann.

- Bei an Long-Covid-Erkrankten sollte insbesondere dann an eine yang-minor-Problematik (Kleines Yang, shaoyang) gedacht werden, wenn eine auffällige Wechselhaftigkeit von Symptomatik und bzw. Temperaturempfinden besteht bzw. wenn Ausmaß und plötzliches Auftreten einer Schwächesymptomatik in Relation zu auslösenden Triggern als unangemessen auffallen.

- In Erweiterung der Angaben in der „Abhandlung über schädigende Kälte (algor laedens)“" (Shanghan lun), nach denen üblicherweise nach einigen Tagen bis etwa zwei Wochen nach dem ursprünglichen Infekt-Ereignis mit einem yang-minor-Syndrom (Kleines Yang, shaoyang) gerechnet wird, können diese nach Erfahrungen des Autors auch nach sehr viel längerer Zeit (länger als ein Jahr danach) noch eine Rolle spielen und erfolgversprechend mit Modifikationen des „Kleinen Bupleurum-Dekokts“ (Xiao chaihu tang) behandelt werden.

- Vorsicht bei Erkrankten mit arteriellem Hypertonus und Kopfschmerzen durch emporschlagendes yang hepatici (Yang des Fk „Leber“, ganyang).

- Wechselndes Temperaturempfinden bei tardi (,verlangsamten“, chi) und depleti („erschöpften“, xu), mersi (,untergetauchten“, chen) oder superficiales (,oberflächlichen“, $f u$ ) Pulsen weist auf einen calor falsus (,falsche Hitze“, jiare) hin und stellt eine Kontraindikation für das „Kleine Bupleurum-Dekokt“ (Xiao chaihu tang) dar.

- Bei Erkrankten mit gravierender depletio yin (energetische Schwäche des Yin, yinxu) ist die Rezeptur wegen der Möglichkeit eines aufsteigenden ardors (,Glut“, huo) nicht oder nur mit äußerster Vorsicht anzuwenden.

\section{Fallbeispiel 1}

Eine 55-jährige Patientin ohne wesentliche Vorerkrankungen wurde durch ein von einer Reise heimkehrendes Familienmitglied mit SARS-CoV-2 infiziert. Die Akutsymptomatik bestand vorwiegend in Schwäche, Dyspnoe und Fieber über zwei Wochen. Während dieser Zeit befand sich die Patientin ohne weitere Therapie in häuslicher Quarantäne. Die Patientin stellte sich dann nach mittlerweile monatelanger Arbeitsunfähigkeit erstmalig vor und klagte über anhaltende Schwäche, schnelle und gravierende Erschöpfung bzw. Ermüdbarkeit auch nach leichten Anstrengungen, Defizite bei Konzentration und Merkfähigkeit sowie über Wortfindungs- 
störungen und anhaltende depressive Verstimmung. Auffällig waren häufig wechselndes Temperaturempfinden und ein zeitweiliger bitterer Mundgeschmack. Unter der Annahme eines Long-Covid-Syndroms im Rahmen einer Dysharmoniestörung des yang minor (Kleines Yang, shaoyang) wurde folgende Modifikation des „Kleinen Bupleurum-Dekokts“ (Xiao chaihu tang) verordnet:

- Bupleuri radix (Chaihu)

- Scutellariae radix (Huangqin)

- Pinelliae rhizoma (Banxia)

- Zingiberis rhizoma recens (Shengjiang)

- Codonopsis radix (Dangshen)

- Glycyrrhizae radix (Gancao)

- Jujubae fructus (Dazao)

- Acori rhizoma (Shichangpu)

- Polygalae radix (Yuanzhi)

- Atractylodis rhizoma (Cangzhu)

- Magnoliae cortex (Houpo)

Die Rezeptur wurde mit leichten Abänderungen über insgesamt acht Wochen eingenommen. Nach kurzer anfänglicher Schleimexpektoration erfolgte in dieser Zeit eine komplette Restitution der kognitiven Fähigkeiten der Patientin, und die Kräfte wurden so weit wiederhergestellt, dass mit einer schrittweisen Arbeitswiedereingliederung begonnen werden konnte. Nach Einnahme eines modifizierten „Dekokts der sechs Edlen“ (Liu junzi tang, s. oben) über weitere zwei Monate fühlte sich die Patientin emotional und kräftemäßig voll wiederhergestellt und konnte ihrer Arbeit ohne Einschränkungen wieder nachgehen.

\section{Long-Covid bei Kindern und Jugendlichen}

Während schwere Verläufe von Covid-19 bei Kindern und Jugendlichen ohne risikobehaftete Vorerkrankungen sehr selten sind, bestand längere Zeit noch Unklarheit über die Relevanz von Long-Covid-Erkrankungen in diesen Altersgruppen. Neuere Studien bzw. Vorveröffentlichungen von Universitäten in Zürich (Radtke et al. 2021), Melbourne (Say et al. 2021), Großbritannien (Duncan et al. 2021) legen nahe, dass Kinder im Schulalter nach SARS-CoV-2Infektionen nur in seltenen Fällen Symptome aufweisen, die Long-Covid zugeordnet werden könnten. Nach Ablauf eines Beobachtungszeitraum von zwei bis drei Monaten nach Infektion waren Symptome in diesen Studien nicht häufiger als bei Kindern in Kontrollgruppen ohne nachgewiesene SARS-CoV-2-Infektion. Vielmehr wurde u. a. auch durch Arbeiten aus Deutschland (Dresden (Armann et al. 2021) bzw. Hamburg/Berlin, Multicenter (Ravens-Sieberer et al. 2021)) bestätigt, dass einerseits Kinder und Jugendliche offenbar sehr selten an anhaltenden Beschwerden im Sinne einer Long-Covid-Erkrankung leiden und diese in aller Regel nach drei Monaten abgeklungen sind. Andererseits jedoch wurden bei infizierten und nicht infizierten Kindern gehäuft Störungen beobachtet, die darauf hinweisen, dass psychische, psychosomatische und psychosoziale Störungen und Probleme als Folge von sozialer Isolation z.B. durch KiTa-Schließungen und Fehlen von Präsenzunterricht in den Schulen sowie familiäre Belastungen durch Lockdown-Maßnahmen die dominierenden Leiden in dieser Altersgruppe zu sein scheinen (,Long-Pandemic Syndrome“" (Armann et al. 2021)).

Einen Sonderfall stellt das seltene „Pediatric Inflammatory Multisystem Syndrome“ (PIMS) dar, das sich ca. zwei bis vier Wochen nach einer (oft asymptomatischen) SARSCoV-2-Infektion entwickeln kann, am häufigsten bei Jungen im Grundschulalter aufzutreten scheint und typischerweise durch plötzlich auftretendes Fieber, reduzierten Allgemeinzustand sowie unterschiedliche Organbeteiligungen (vorwiegend betroffen: Herz-Kreislauf-System, Gastrointestinaltrakt, Haut und Schleimhäute) gekennzeichnet ist. Die Erkrankung verläuft meist so schwer, dass eine stationäre, oft auch intensivmedizinische Behandlung notwendig wird. Während aus den USA Todesfälle in Zusammenhang mit PIMS-Erkrankungen berichtet werden, sind in Deutschland glücklicherweise bisher keine derartig schlimmen Verläufe dokumentiert worden.

In einer britischen Studie, in der Folgesymptome bei Kindern untersucht wurden, die eine stationär behandlungsbedürftige PIMS-Erkrankung durchgemacht hatten, wurde sechs Monate nach Abklingen der Akutsymptomatik in fast allen Fällen ein komplettes Abklingen der Beschwerden beobachtet (Moshal et al. 2021).

Aus Sicht der Chinesischen Medizin ist bei nach SARSCoV-2-Infektion persistierenden Beschwerden von jüngeren Kindern bis zum Grundschulalter am ehesten an Störungen der „Mitte“ mit depletio (energetische Schwäche, $x u$ ) des Qi und Akkumulation von humor („Feuchtigkeit“", shi) bzw. pituita (,Schleim“, tan) zu denken, während bei älteren Kindern auch eine yang-minor-Dysharmonie (Kleines Yang, shaoyang) in Betracht zu ziehen ist.

\section{Fallbeispiel 2}

Ein 12-jähriges Mädchen entwickelte ca. vier Wochen nach einer möglichen Infektion mit SARS-CoV-2 (initial bei minimaler Atemwegssymptomatik kein PCR durchgeführt, späterer PCR negativ, jedoch Nachweis von SARS-CoV-2IgM und -IgG) eine beinbetonte Akrozyanose mit Schmerzen der Finger und Zehen sowie milde Oberbauchschmerzen mit Unwohlsein, begleitet von schnell wechselndem Temperaturempfinden. Unter der Annahme einer yang-minor-Störung (Kleines Yang, shaoyang) mit Aspekten von Xue-Stasen wurde als Grundrezept das „Kleine Bupleu- 
rum-Dekokt“" (Xiao chaihu tang, s. oben) verordnet, ergänzt durch Spatholobi caulis et radix (Jixueteng), Achyranthis bidentatae radix (Niuxi) und Olibanum (Ruxiang). Nach zweiwöchiger Einnahme waren die Beschwerden in den Extremitäten komplett rückläufig, und bei noch geringen abdominellen Beschwerden und wechselndem Temperaturempfinden wurde das Basisrezept ohne die das Xue dynamisierenden Arzneien noch für weitere zwei Wochen bis zur Beschwerdefreiheit eingenommen.

\section{Schlussbemerkung}

Die Chinesische Medizin kann für die zunehmende Zahl von an Long-Covid-Erkrankten mit respiratorischen Symptomen, mit sensorisch-mentalen Defiziten sowie an Chronic-Fatigue-Syndrom (CFS) Leidenden, für die im Bereich der etablierten westlichen Medizin wirksame Therapieansätze bisher nicht in Sicht sind, sehr effektive Behandlungskonzepte bereitstellen.

\section{Einhaltung ethischer Richtlinien}

Interessenkonflikt C. Thede erklärt, dass er sich bei der Erstellung des Beitrags von keinen wirtschaftlichen Interessen leiten ließ und dass keine potenziellen Interessenkonflikte vorliegen.

Ethische Standards Dieser Beitrag beinhaltet keine Studien an Menschen oder Tieren. Der Verlag erklärt, dass die inhaltliche Qualität des Beitrags von zwei unabhängigen Gutachtern geprüft wurde. Werbung in dieser Zeitschriftenausgabe hat keinen Bezug zur CME-Fortbildung. Der Verlag garantiert, dass die CME-Fortbildung sowie die CME-Fragen frei sind von werblichen Aussagen und keinerlei Produktempfehlungen enthalten. Dies gilt insbesondere für Präparate, die zur Therapie des dargestellten Krankheitsbildes geeignet sind.

\section{Literatur}

Armann J, et al. Mental health of adolescents in the pandemic: longCOVID19 or long-pandemic syndrome? medRxiv. 2021; https:// doi.org/10.1101/2021.05.11.21257037. preprint.

Baig AM. Chronic COVID syndrome: need for an appropriate medical terminology for long-COVID and COVID long-haulers. J Med Virol. 2020; https://doi.org/10.1002/jmv.26624.

Carfi A, Bernabei R, Landi F, et al. Persistent symptoms in patients after acute COVID-19. JAMA. 2020;324(6):603-5. https://doi. org/10.1001/jama.2020.12603.

Datta SD, Talwar A, Lee JT. A proposed framework and Timeline of the spectrum of disease Due to SARS-coV-2 infection: illness beyond acute infection and public health implications. JAMA. 2020; https://doi.org/10.1001/jama.2020.22717.

Duncan E, et al. Illness duration and symptom profile in a large cohort of symptomatic UK school-aged children tested for SARS-CoV-2. medRxiv. 2021; https://doi.org/10.1101/2021.05. 05.21256649. preprint.

Gold JE, Okyay RA, Licht WE, Hurley DJ. Investigation of long COVID prevalence and its relationship to Epstein-Barr virus reactivation. Pathogens. 2021;10:763. https://doi.org/10.3390/ pathogens 10060763 .
Hempen C-H, Fischer S, Hummelsberger J, Koch A, Leonhardy H, Nögel R, Thede C, Wullinger M. Leitfaden Chinesische Rezepturen. München: Elsevier; 2006.

Manal S, Barnett J, Brill SE, et al. Long-COVID: a cross-sectional study of persisting symptoms, biomarker and imaging abnormalities following hospitalisation for COVID-19. Thorax. 2020;76(4):396-8. https://doi.org/10.1136/thoraxjnl2020-215818.

Moshal K, et al. 6-month multidisciplinary follow-up and outcomes of patients with paediatric inflammatory multisystem syndrome (PIMS-TS) at a UK tertiary paediatric hospital: a retrospective cohort study. Lancet Child Adolesc Health. 2021;2021(5):473-82.

National Health Commission of the PRC, National Administration of Traditional Chinese Medicine of the PRC. Guidance for corona virus disease 2019-prevention, control, diagnosis and management. 7. Aufl. Beijing: People's Medical Publishing House; 2020.

Radtke T, Ulyte A, Puhan M, Kriemler S. Long-term symptoms after SARS-CoV-2 infection in school children: population-based cohort with 6-months follow-up. medRxiv. 2021; https://doi.org/10. 1101/2021.05.16.21257255. preprint.

Ravens-Sieberer U, et al. Quality of life and mental health in children and adolescents during the first year of the COVID-19 pandemic: Results of a two-wave nationwide population-based study. SSRN Journal. 2021; https://doi.org/10.2139/ssrn.3798710.

RKI. Täglicher Lagebericht des RKI zur Coronavirus-Krankheit2019 (COVID-19). 12.12.2020 - AKTUALISIERTER STAND FÜR DEUTSCHLAND. 2020. https://www.rki.de/DE/Content/ InfAZ/N/Neuartiges_Coronavirus/Situationsberichte/Dez_2020/ 2020-12-12-de.pdf?_blob=publicationFile. Zugegriffen: 13. Dez. 2020.

Say D, Crawford N, McNab S, Wurzel D, Steer A, Tosif S. Post-acute COVID-19 outcomes in children with mild and asymptomatic disease. Lancet Child Adolesc Health. 2021;5(6):e22-e3.

Scheid V, Bensky D, Ellis A, Barolet R. Chinese herbal medicine. Formulas \& strategies. 2. Aufl. Seattle: Eastland Press; 2009.

Sollini M, Ciccarelli M, Cecconi $\mathrm{M}$, et al. Vasculitis changes in COVID-19 survivors with persistent symptoms: an [18F]FDGPET/CT study. Eur J Nucl Med Mol Imaging. 2020; https://doi. org/10.1007/2Fs00259-020-05084-3.

Thede C. Indikationen für chinesische Arzneimitteltherapie bei Covid19-Erkrankungen. Z Komplementärmed. 2020a;2020(3):18.

Thede C. Mögliche Therapiestrategien bei Covid-19-Erkrankungen mit chinesischen Arzneimitteln. Chin Med. 2020b;2020(2):55-78.

Thede C. COVID-19 - Therapiemöglichkeiten mit chinesischen Arzneimitteln in der Akutphase und Rekonvaleszenz. Dtsch Z Akupunkt. 2020c;63(4):208-13. https://doi.org/10.1007/s42212-02000316-x.

Thede C. Post-COVID-Syndrom: Behandlung von Residuen nach SARS-CoV-2-Infektionen mit Chinesischer Arzneimitteltherapie. Z Komplementärmed. 2021;2021(1):46-50. https://doi.org/ 10.1055/a-1332-0581.

Thede C, et al. Mögliche Therapiestrategien von COVID-19 Erkrankungen mit chinesischen Behandlungsmethoden. 2020. https:// www.tcm.edu/tcm-covid-19/chinesische-arzneimitteltherapie/ (Erstellt: 25. März 2020). Zugegriffen: 25.06.2021.

Zhao J, Flanagan C. Approaching covid-19 as damp toxin. Lantern. 2020;17(2a):16-23.

Zhao Z-H, et al. Analysis of traditional Chinese medicine diagnosis and treatment strategies for COVID-19 based on "the diagnosis and treatment program for Coronavirus disease-2019" from Chinese authority. Am J Chin Med. 2020;48(5):1035-49. https://doi. org/10.1142/S0192415X20500500.

Zhong LLD, et al. Potential targets for treatment of Coronavirus disease 2019 (COVID-19): a review of Qing-Fei-Pai-Du-tang and its major herbs. Am J Chin Med. 2020;48(5):1051-71. https://doi. org/10.1142/S0192415X20500512. 
Christian Thede (geb. 1960), Arzt für Allgemeinmedizin, Praxis für Chinesische Medizin seit 1993, Dozent für Chinesische Medizin bei der SMS, ehemaliger Dozent der Ärztekammer Hamburg sowie ehemaliger Lehrbeauftragter an der Universität Witten-Herdecke, Mitglied im Prüfungsausschuss Akupunktur der Ärztekammer Schleswig-Holstein, Mitautor des Buches Leitfaden Chinesische Rezepturen. 
Zu den Kursen dieser Zeitschrift: Scannen Sie den QR-Code oder gehen Sie auf www.springermedizin.de/cme

? Als wesentliches infektiöses Pathogen bei einer Infektion mit SARS-CoV-2 gilt nach übereinstimmender Auffassung

O calor humidus (,Feuchtigkeit-Hitze“, shire) im unteren Calorium (unterer Wärmebereich, xiajiao)

O algor-Blockade (,Kälte“, han) der extima (Oberfläche, biao)

O depletio qi (energetische Schwäche des Qi, qixu) der oo. pulmonalis et lienalis (Fk „Lunge“ und „Milz“, fei pi)

O epidemisches humor-Toxisches (,Feuchtigkeit“-Toxisches, shidu)

O Dysharmonie des yin minor (Kleines Yin, shaoyin)

? Zur Behandlung einer depletio $q i$ (energetische Schwäche des Qi, qixu) der oo. pulmonalis et lienalis (Fk „Lunge“" und „Milz“, fei pi) nach einer COVID-19-Erkrankung ist folgende Rezeptur geeignet:

O „Das Dekokt gegen die vier Kontravektionen" (Sini tang)

O „Dekokt der vier Bestandteile“ (Siwu tang)

O „Rehmannia-Pille mit sechs Geschmacksrichtungen" (Liuwei dihuang wan)

O „Pulver gegen die vier Gegenläufigkeiten“( (Sini san)
„Dekokt der sechs Edlen“ (Liu junzi tang)

? Zur Therapie einer depletio yin (energetische Schwäche des Yin, yinxu) des o. pulmonalis (Fk ,Lunge“, fei) nach einer COVID-19-Erkrankung ist folgende Arznei indiziert:

O Ophiopogonis radix (Maimendong)

O Pinelliae rhizoma (Banxia)

O Rehmanniae radix (Shengdihuang)

O Mori cortex (Sangbaipi)

Mori fructus (Sangshen)

? Welche Arznei kann bei respiratorischen Störungen nach COVID-19-Erkrankungen in geeigneten Kombinationen sowohl bei pituita algida (,KälteSchleim", hantan) als auch bei pituita calida (,Hitze-Schleim“", retan) eingesetzt werden?

O Sinapis albae semen (Baijiezi)

O Pinelliae rhizoma (Banxia)

O Bambusea concretio silicea (Tianzhuhuang)

O Typhonii rhizoma praeparata (Baifuzi)

O Ostreae concha (Muli)
? Zur Behandlung von Xue-Stasen im Bereich des Thorax nach Infektionen mit SARS-CoV-2 ist folgende Arznei besonders geeignet:

O Spatholobi caulis et radix (Jixueteng)

O Scutellariae radix (Huangqin)

O Salviae miltiorrhizae radix (Danshen)

O Glycyrrhizae radix (Gancao)

O Leonuri herba (Yimucao)

? Eine sowohl bei Long-COVIDErkrankungen als auch bei chronischen EBV-Infektionen häufig vorkommende energetische Entgleisung ist

O Dysharmonie des yin minor (Kleines Yin, shaoyin)

O Stagnation durch algor („,Kälte“, han) in $\operatorname{der} c$. hepatica (Leitbahn des Fk „Leber“, ganjing)

O Dysharmonie des yang minor (Kleines Yang, shaoyang)

O calor humidus (,,Feuchtigkeit-Hitze“, shire) im o. vesicalis (Fk „Blase“, pangguang)

O Dysharmonie der Wandlungsphasen Feuer und Wasser (oo. cardialis et renalis, $\mathrm{Fk}$ „Herz“ und „Niere“, xin shen)

\section{Informationen zur zertifizierten Fortbildung}

Dieser CME-Kurs wurde von der Bayerischen Landesärztekammer in der Kategorie I (tutoriell unterstützte Online-Maßnahme) zur zertifizierten Fortbildung frei gegeben und ist damit auch für andere Ärztekammern anerkennungsfähig. Je nach Bearbeitungsdauer eines Beitrags werden 2 bis 4 Punkte vergeben (siehe im Anschluss an die letzte Frage).
Bei inhaltlichen Fragen erhalten Sie beim Kurs auf SpringerMedizin.de/CME tutorielle Unterstützung. Bei technischen Problemen erreichen Sie unseren Kundenservice kostenfrei unter der Nummer (0800) 7780777 oder per Mail unter kundenservice@springermedizin.de.
Hinweise zur Teilnahme:

- Die Teilnahme an dem zertifizierten Kurs ist nur online auf www.springermedizin.de/cme möglich.

- Der Teilnahmezeitraum beträgt 12 Monate. Den Teilnahmeschluss finden Sie online beim Kurs.

- Die Fragen und ihre zugehörigen

Antwortmöglichkeiten werden

online in zufälliger Reihenfolge

zusammengestellt.

\footnotetext{
- Pro Frage ist jeweils nur eine Antwort zutreffend.

- Für eine erfolgreiche Teilnahme müssen 70\% der Fragen richtig beantwortet werden.

- Teilnehmen können Abonnenten dieser Fachzeitschrift und e.Med-Abonnenten.
} 
? Zwei synergistisch wirkende Arzneien aus der in der, ,Abhandlung über schädigende Kälte (algor laedens)“" (Shanghan lun) genannten Hauptrezeptur zur Behandlung einer Dysharmonie des yang minor (Kleines Yang, shaoyang) sind

O Scutellariae radix (Huangqin) und Coptidis rhizoma (Huanglian)

O Bupleuri radix (Chaihu) und Paeoniae radix lactiflora (Baishao)

O Cinnamomi cassiae ramulus ( Guizhi) und Paeoniae radix lactiflora (Baishao)

O Bupleuri radix (Chaihu) und Astragali radix (Huangqi)

O Bupleuri radix (Chaihu) und Scutellariae radix (Huangqin)

? In welchen Situationen sollte von der Verwendung des ,Kleinen Bupleurum-Dekokts" (Xiao chaihu tang) abgesehen werden?

O bei gleichzeitiger Long-CovidSymptomatik und einer EBV-Infektion

O bei Verdacht auf mögliche persistierende Agenzien

O bei Symptomen und Zeichen eines calor falsus (,falsche Hitze“, jiare)

O bei wechselndem Temperaturempfinden und bitterem Mundgeschmack

O bei chronischen und protrahierten Infekten

? Das yang minor (Kleines Yang, shaoyang) hat im Kontext der „Abhandlung über schädigende Kälte (algor laedens)“ (Shanghan lun) keine direkte oder indirekte Bedeutung für

O im Verlauf von Infekten auftretende Blockaden und depletio (energetische Schwäche, $x u$ ) der "Mitte“

O die harmonische Funktion des Öffnens und Schließens im Bereich des yang maior (Großes Yang, taiyang) und des splendor yang
(Überstrahlung des Yang, yangming)

O das Auftreten eines kurzfristig wechselnden Temperaturempfindens

O die Speicherung und Freisetzung des ignis ministri (,,ministerielles Feuer", minghuo)

O das Auftreten eines bitteren Mundgeschmacks

? Welche der folgenden Arzneien zur Behandlung von depletio yin (energetische Schwäche des Yin, yixu) bzw. Säfte-Verlust ist bei Frauen im gebärfähigen Alter kontraindiziert?

O Trichosanthis radix (Tianhuafen)

O Glehniae radix (Beishashen)

O Ophiopogonis radix (Maimendong)

O Polygonati odorati rhizoma (Yuz$h u)$

O Panacis quinquefolii radix (Xiyangshen)

Dieser CME-Kurs wurde von der Bayerischen Landesärztekammer mit 2 Punkten in der Kategorie I (tutoriell unterstützte Online-Maßnahme) zur zertifizierten Fortbildung freigegeben. 